\title{
AUTOMATION OF LIQUID FILLING AND MIXING/AGITATING SEQUENTIALLY WITH PRE-DEFINED VOLUME USING PLC
}

\author{
Rashmi Jakati ${ }^{1}$, Anand Unnibhavi ${ }^{2}$ \\ ${ }^{1}$ Department of ECE, Basaveshwar Engineering College, Bagalkot, Karnataka, India \\ ${ }^{2}$ Department of ECE, Basaveshwar Engineering College, Bagalkot, Karnataka, India
}

\begin{abstract}
This paper deals with the "Automated liquid filling and mixing/agitating sequentially with the pre-defined volume using PLC". This kind of automation is necessary in Pharmaceutical Industries mainly in Syrup manufacturing Industries where the amount of different ingredients to be added must be precise and accurate. It also increases the productivity rate and reduces the cost. Codeine Linctus, a cough syrup is taken as the example which consists of totally six liquid ingredients with proportionate volume. Programmable Logic controller (PLC) is used for making the procedure automatic. Proximity sensors, motors, solenoid valves and mechanical agitator are employed in the project. Simulation of particular ladder diagram is carried out using IndraWorks Engineering.
\end{abstract}

Keywords: PLC, Liquid, Filling, Mixing, Agitating, Codeine Linctus

\section{INTRODUCTION}

In the past few years, after the Industrial revolution industries have adopted several automated machines for its purpose. Earlier Industries were much dependent on the manpower. In order to reduce the human intervention automation was adopted. The automation also achieves the productivity rate by increased speed of manufacturing, accuracy and quality of the products by reducing human error. The reduced cost and effective utilization of resources has a greater impact on the world economy. Automation has wider applications in the field of manufacturing industries like concrete industries, beverage industries, paint industries, for milling, grinding, packaging and sorting etc. The main goal of this paper is to adopt automation in the pharmaceutical industries like medicinal syrup manufacturing industries. In case of, pharmaceutical industries the amount of addition of ingredients like acids, sugar-water solution and heating the solution to a particular temperature play a very important role, otherwise leads to harmful chemical reactions. Hence, in order to avoid such bad effects automation is most preferred.

This paper explains mainly about the filling of predefined volume of liquid solutions like purified water, acids, lemon syrup, color agents, and flavors and mix them uniformly using PLC based automation. Preparation of Codeine Linctus, a cough syrup, the appropriate amount of solution filling and mixing is carried out. There are few methods for preparation of syrups like, Solution with heat, Agitation without heat and percolation method. For preparation of Codeine Linctus, the method called Agitation without heat is preferred. The method is best suitable for volatile substances.

\section{INDRALOGIC PLC}

The PLC is programmable device which performs several complicated industrial operations by replacing mechanical timers, counters and relays. It is an industrial computer capable of storing user-oriented instructions to implement control functions such as counting, timing, sequencing, arithmetic data manipulation and communication by making use of programmable memory. Generally, PLC consists of control unit and a set of input and output modules based on different configurations and monitored by SCADA(Supervisory Control and Data Acquisition). The input module includes sensors, push button switch and output module such as motors, conveyor, and solenoid valve which allow PLC to interact with real time systems.

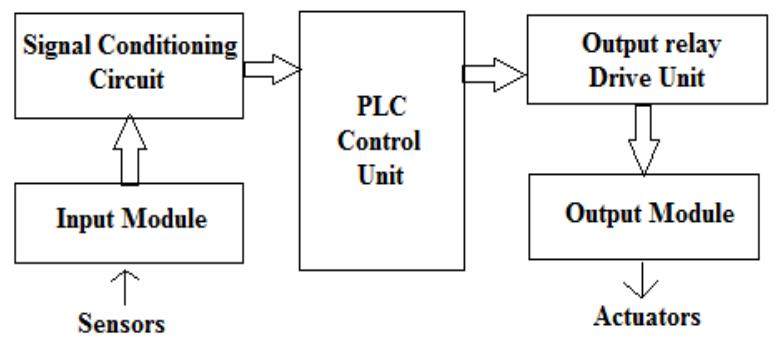

Fig 1: Block Diagram of Basic components of PLC.

The Basic components of PLC are as shown in figure a. Control unit is also called as Central Processing Unit. It retrieves the data sensed by the sensors, decodes, stores and process the data. The processed data is decoded again then sent to the output devices. The sensors include proximity sensors such as capacitive sensor, inductive sensor, ultrasonic and optical sensor etc,. The Input module converts the signal sensed by the sensor to signal that is compatible by the PLC to process. The output of the sensors cannot be given directly to the PLC since, PLC requires $24 \mathrm{~V}$ DC 
supply for its operation. Therefore, Signal conditioning circuits are used. Likewise, Output relay drive unit at the output side is used to convert PLC output voltage of $24 \mathrm{~V}$ DC to $12 \mathrm{~V}$ DC because output devices such as motor, gears, pumps and LED may get damaged with supply of larger voltages.

\section{OBJECTIVE}

To develop an automated sequential liquid filling and agitating with appropriate volume using PLC. This kind of automation is mainly employed in Pharmaceutical industries where the medicinal syrups are prepared. Preparation of Codeine Linctus, a cough syrup is considered in this paper.

\section{METHODOLOGY}

This chapter explains about the automated agitation without heating method for the preparation of Codeine Linctus. This method provides uniform dissolving of volatile substances in the purified water without heating any substance. The automation is done by making use of sensors, solenoid valves, motors, timers, push button switches and conveyor belt. The proximity sensors are used to detect the object. The solenoid valves (Flow control valves) are used for filling pre-defined volume of liquid where timers (Off-delay timers) are used for the appropriate measurement of volume. The motors drive the conveyor belt.

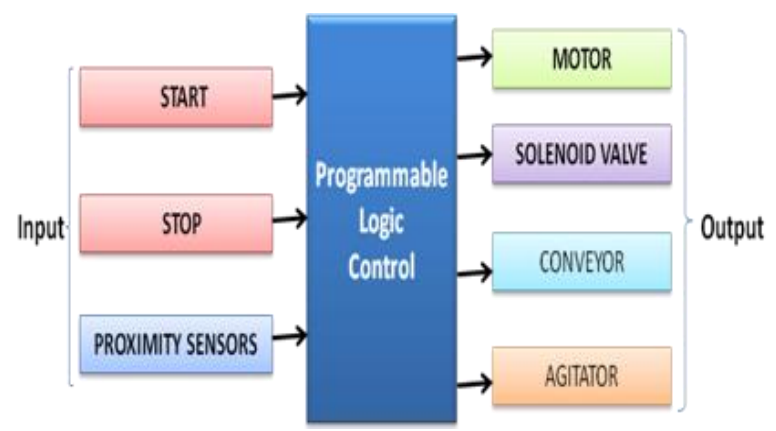

Fig 2: Block diagram of PLC used for Liquid filling and agitating.

The figure 2. shows the block diagram of PLC used in liquid filling and agitating for the preparation of Codeine Linctus. The components used are two Push button switches, seven Proximity sensors, DC Motor, Solenoid valve, Mechanical agitator and Conveyor belt.

The description of the Components used:

1. Push button switch: This is the most common switch used. Momentary and Maintained push button switches are the two types. The momentary switch is activated when pressed and deactivated when released since, it is done using internal spring. The maintained switch is activated when pressed remains activated until it is pressed again. Here we use maintained type of push button switches.

2. Proximity sensor: The sensor detects and sends the signal to the PLC when the object is detected in the operating zone. There are different proximity sensors like inductive, capacitive, ultrasonic and optical proximity sensors. Here we use capacitive proximity sensor which detects both conductive and non-conductive material. The sensor has to detect the glass-lined tank in the present work.

3. DC Motor: The motor requires 12V DC for its operation which is supplied by output relay drive unit. The DC motor is used to drive the conveyor belt which moves in forward direction.

4. Conveyor: The DC motor drives the conveyor to move in a forward direction. It will stop according to the sensor or limit switch position. The conveyor helps to transfer the materials from one station to the other.

5. Solenoid valve: These are flow control valves with electromagnetic plunger which control the flow of fluids or liquids. The input to the valve is given by the PLC. The process considered in this paper uses six Solenoid valves for six different tanks in order to fill the liquid in the container or glass-lined tank. The capacitive sensors are placed below each tank containing different ingredients of the Codeine Linctus. The sensor when detects the glass-lined tank or any container, it sends the signal to the PLC, the conveyor stops simultaneously the solenoid values turns $\mathrm{ON}$ and predefined volume of liquid (Ingredient) flows in to the tank, the volume of the liquid (Ingredient) is decided by making use of timers.

Timers with different preset time for different volume of ingredients are given to the tanks. Timer turns ON when the objected is detected by the sensor, conveyor stops. As soon as the timer turns $\mathrm{ON}$, the solenoid valve turns $\mathrm{ON}$ and Particular ingredients is filled in the tank. The timer turns OFF after the set time is over, solenoid valve goes OFF conveyor starts to move in forward direction. The process repeats until all the ingredients are filled in the tank. The mixing or agitating of the entire solution is to be done, so the sensor is placed at the agitator and mechanical agitator is used for the purpose.

1. Mechanical Agitator: The mixing or agitating of the entire solution is carried out. The sensor is placed at the agitator, once the sensor detects the container the mechanical agitator starts stirring the entire solution uniformly for pre-defined time which is set by the timer. The input voltage $(12 \mathrm{~V} \mathrm{DC})$ to the agitator is given by the output- relay conditioning circuit.

2. Timer: It is one of the PLC ladder functions which allows the PLC to perform certain timing operations based on a preset clock. Timers are of three types,

ON delay timer: When the input to the timer goes high, it gets activated only after the preset time is elapsed and gets deactivated when the input goes low.

OFF delay timer: When the input to the timer goes high, the timer is activated. As soon as input goes low the timer gets deactivated after the preset time is elapsed. 
Pulse timer: When the input to the timer goes high, the timer is activated remains activated till the preset time is elapsed and after that again resets even if the input is high. OFF delay timer is used in case of liquid filling and agitating. The input to the timer is given by proximity sensor.

\section{PROCESS DESCRIPTION}

The basic process of automated liquid filling and agitating is as shown in the fig 3. Preparation of the Codeine Linctus involves filling of ingredients one by one in the glass-lined tank with specified volume and mixing them thoroughly. The steps for the preparation is given sequentially below.

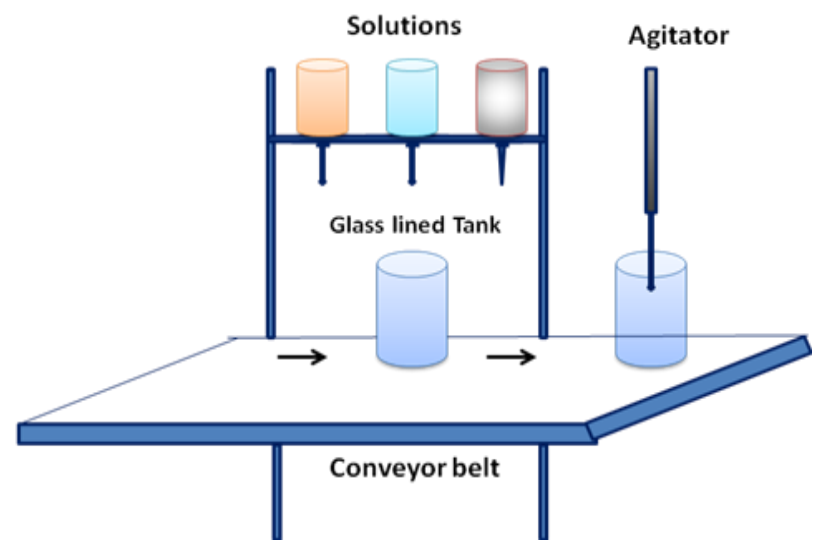

Fig 3: Filling and mixing of medicinal liquids for the preparation of Codeine Linctus solution

\subsection{Detection of Glass-Lined Tank}

The container that we use here is of Glass-lined. The capacitive proximity sensor placed below each tank detects the presence of the container and sends the signal to the PLC. As soon as the sensor detects the conveyor stops moving. Depending on the output of the sensor filling and mixing/agitating operation takes place.

\subsection{Filling the Liquid Ingredients into the Tank}

The ingredients to be added are placed in separate tanks as shown in the Fig c. There are six different ingredients in the liquid form and table 1.gives the composition of the Codeine Linctus syrup. Solenoid valve or the flow control valves are attached to the tanks to release the liquid into the container. The container is placed on the conveyor belt, as soon as the motor turns on the conveyor starts to move in forward direction, when it reaches the first ingredient tank the sensor detects the container the solenoid valve turns on and release the liquid into the tank and once the solenoid valves turns OFF the conveyor starts to move and again when container reaches to the next tank, the valve turns on release the ingredient and the cycle repeats until six different liquids are released into the container. At last the sensor placed on the mechanical agitator detects the container and agitator starts to stir the liquids placed in the container until the preset time of the timer is elapsed for the uniform distribution of all the ingredients.

\subsection{Pre-defined Volume}

The appropriate volume of the ingredients to be released into the container is decided by the timer. Based on different quantity of liquid ingredients the preset time of the timers is set. The head of the pipe of the tanks containing different ingredient liquids is assumed to be of $50 \mathrm{~mm}$ which releases liquid at the rate of 0.8 liter/sec.

\subsection{Mixing of Liquids}

Six different liquids filled in the container must uniformly mixed, for which a mechanical agitator is used. The proximity sensor placed at the agitator senses the presence of the container and conveyor stops, agitator starts to stir for some pre-defined time and the OFF delay timer with preset time decides duration of mixing. Hence, the Codeine Linctus syrup solution is ready.

Table 1: Composition of Codeine Linctus.

\begin{tabular}{|l|l|}
\hline Ingredients & $\begin{array}{l}\text { Volume of the } \\
\text { ingredients }\end{array}$ \\
\hline Codeine Phosphate & $3 \mathrm{grams}$ \\
\hline Lemon Syrup & $200 \mathrm{ml}$ \\
\hline Benzoic acid solution & $20 \mathrm{ml}$ \\
\hline Chloroform Spirit & $20 \mathrm{ml}$ \\
\hline Purified water & $20 \mathrm{ml}$ \\
\hline Compound Tartrazine & $10 \mathrm{ml}$ \\
\hline
\end{tabular}

\section{LADDER DIAGRAM}

Ladder diagram is the graphical representation of PLC program of relay logic hardware circuits. The diagram has two vertical rails and many horizontal rungs running between the rails. The inputs (push button switch and sensors) and outputs (DC motor, agitator, solenoid valve, and conveyor) are placed on the rungs. The output is driven by the inputs as per the requirement. The ladder diagram for the liquid filling and agitating is as given the Figure d.

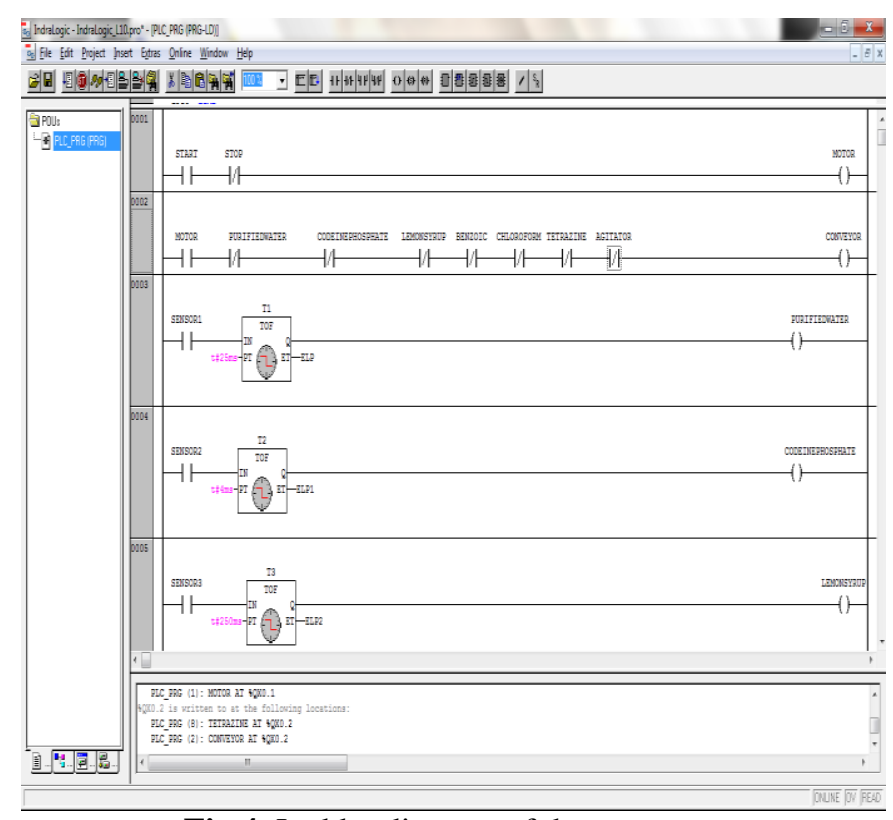

Fig 4: Ladder diagram of the process. 


\section{SIMULATION RESULTS}

The ladder diagram is simulated using Indralogic engineering works. The simulation results are shown in the Figure 5.1.

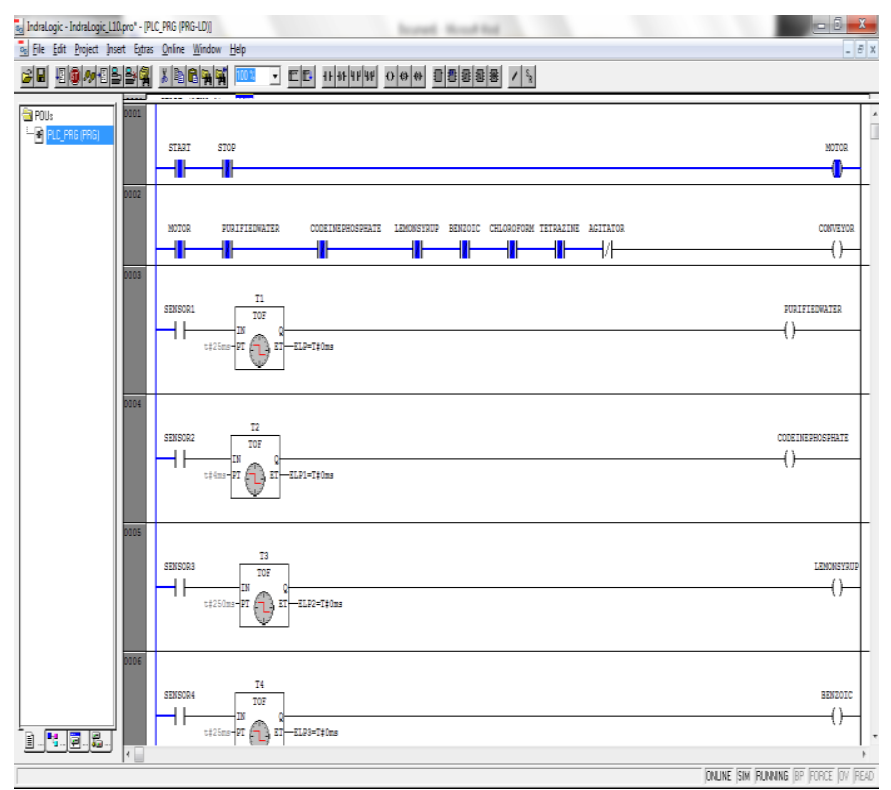

Fig 5.1

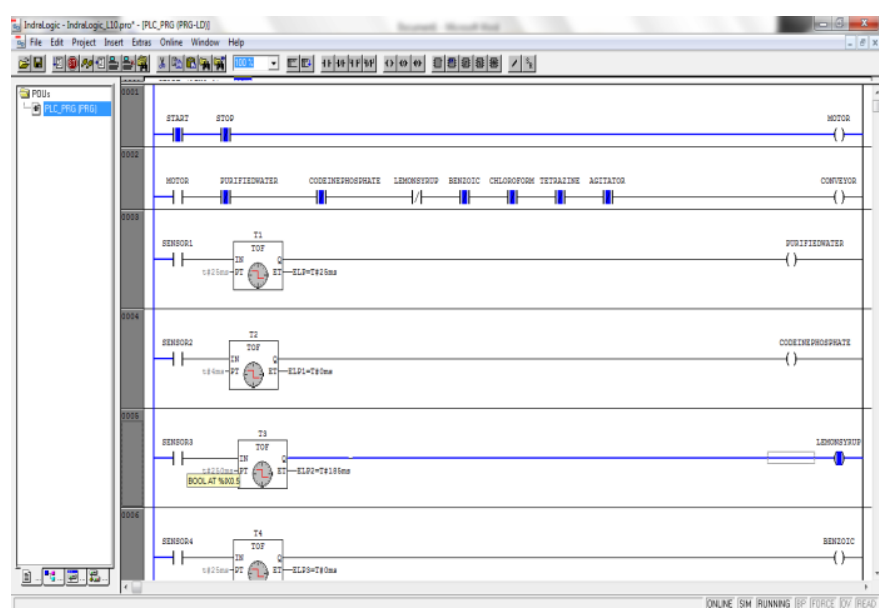

Fig 5.2

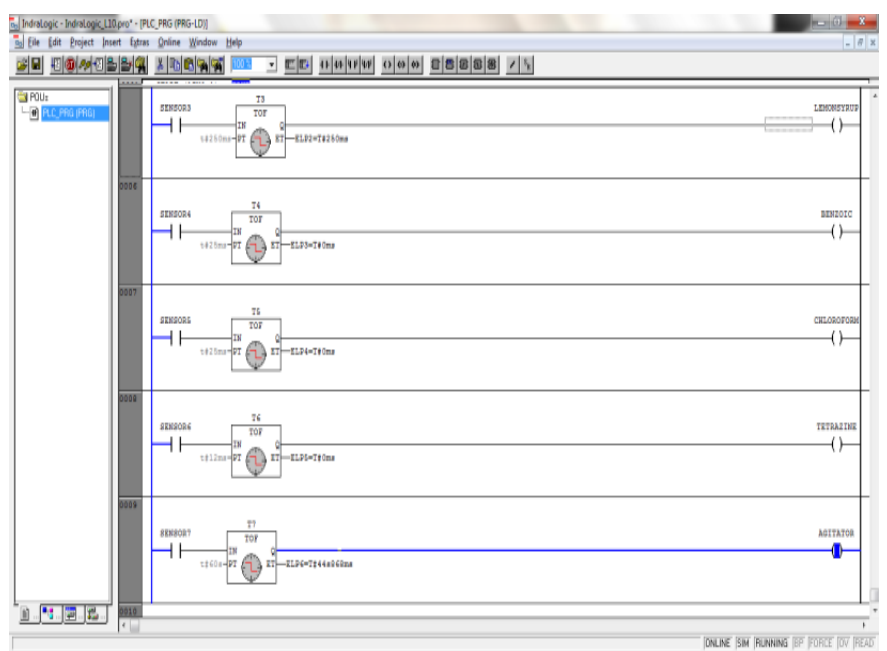

Fig 5.3

\section{CONCLUSION}

The main objective of the project was to automate the filling of ingredients with appropriate volume sequentially and mixing them thoroughly. PLC ladder diagram for liquid filling and mixing is simulated. The project works effectively avoiding unnecessary wastage of liquids and also meets the demands of larger productivity rate and lesser cost.

\section{REFERENCES}

[1]. Mihir Panchal, Aashish Panaskar, Prof. Lalit Kumar," PLC Based Liquid Filling and Mixing", International Journal of Electrical and Electronics Research, ISSN: 23486988 , Vol. 3, Issue 1.

[2]. D.Baladhandabany,S.Gowtham, T.Kowsikkumar, P.Gomathi, " PLC Based Automatic Liquid Filling System", International Journal of Computer Science and Mobile Computing, Vol.4 Issue.3, March- 2015.

[3]. Philip Babatunde OSOFISAN, "Fuzzy Logic Control of the Syrup Mixing Process in Beverage Production “,Leonardo Journal of Sciences, ISSN: 1583-0233.

[4]. T.Kalaiselvi , R.Praveena, "PLC Based Automatic Bottle Filling and Capping System With User Defined Volume Selection", International Journal of Emerging Technology and Advanced Engineering”, (ISSN: 2250-2459 )2012.

[5]. Sagar Patil, Aditi Kurhade, Prof. H.P. Chaudhari, "Juice Flow Stabilization Using PLC and Simulating With SCADA", International Journal of Engineering and Technical Research (IJETR), ISSN: 2321-0869, Volume-3, Issue-3, March.

[6]. Rukkumani V, Khavya S, Madhumithra S., Nandhini Devi B,"Chemical Process Control In Sugar Manufacturing Unit", International Journal of Advances in Engineering \& Technology, Jan. 2014, ISSN: 2231-1963. 